\title{
FAST DIFFERENTIAL EMISSION MEASURE INVERSION OF SOLAR CORONAL DATA
}

\author{
Joseph Plowman, Charles Kankelborg, and Petrus Martens \\ Montana State University, Bozeman, MT 59717, USA \\ Received 2012 October 12; accepted 2013 May 1; published 2013 June 7
}

\begin{abstract}
We present a fast method for reconstructing differential emission measures (DEMs) using solar coronal data. The method consists of a fast, simple regularized inversion in conjunction with an iteration scheme for removal of residual negative emission measure. On average, it computes over $1000 \mathrm{DEMs} \mathrm{s}^{-1}$ for a sample active region observed by the Atmospheric Imaging Assembly (AIA) on the Solar Dynamics Observatory, and achieves reduced chi-squared of order unity with no negative emission in all but a few test cases. The high performance of this method is especially relevant in the context of AIA, which images of order one million solar pixels per second. This paper describes the method, analyzes its fidelity, compares its performance and results with other DEM methods, and applies it to an active region and loop observed by AIA and by the Extreme-ultraviolet Imaging Spectrometer on Hinode.
\end{abstract}

Key words: methods: numerical - Sun: corona - techniques: image processing

Online-only material: color figure

\section{INTRODUCTION}

The Sun's outer atmosphere, the corona, is a hot $\left(\sim 10^{6} \mathrm{~K}\right)$, highly magnetized, dynamic plasma, with spatial scales ranging from the electron gyroradius of less than $1 \mathrm{~cm}$ to the solar diameter of over $10^{11} \mathrm{~cm}$. As such, it is one of the most fruitful objects for the study of astrophysical plasmas. One of the most fascinating puzzles of the corona is how it is heated to temperatures $\sim 1000$ times those of the underlying photosphere. It is believed that this process is magnetic in nature, but the details (e.g., the spatial distribution of heating and whether it is continuous or impulsive) are not well understood. One important tool for understanding these details is the reconstruction of solar temperature distributions called "differential emission measures" (DEMs) from spectral images of the Sun.

The DEM, $\mathcal{E}(T)$, characterizes coronal emission at a given temperature, ${ }^{1}$ and is defined in terms of the densities, $n(l)$, along the line of sight so that $\int \mathcal{E}(T) d T \equiv \int n^{2}(l) d l$ (column emission convention). Observed intensities, $g_{n}$, are given by integrating the instrument response, $R_{n}(T)$, against the DEM being observed:

$$
g_{n}=\int R_{n}(T) \mathcal{E}(T) d T
$$

Algorithms which reconstruct these temperature distributions must be very fast if they are to process the volume of data produced by modern solar observatories, or resolve the dynamics and fine spatial scales believed to be involved in coronal heating. For example, matching the real-time data rate of the Atmospheric Imaging Assembly (AIA; Lemen et al. 2012) requires computation of over $10^{5} \mathrm{DEMs} \mathrm{s}^{-1}$.

There are a number of existing algorithms for reconstructing DEMs from solar image data, but they are far too slow to meet this requirement. One widely used method is the PINTofALE code (Kashyap \& Drake 1998), which employs a Markov Chain Monte Carlo (MCMC) search taking seconds or minutes

\footnotetext{
1 For most spectral lines; lines corresponding to forbidden transitions have more complicated density sensitivity, but that is beyond the scope of this paper. Interested readers may consult del Zanna \& Mason (2005).
}

to compute a DEM. Another method, which is considered relatively fast and was recently applied to AIA data by Hannah $\&$ Kontar (2012), computes $\sim 4$ DEMs s ${ }^{-1}$ (an updated version with substantially improved performance has recently been made available at http://www.astro.gla.ac.uk/iain/demreg/map/; we use this version in our comparisons).

We present a fast, iterative, regularized method for inferring DEMs using data from solar imagers, such as AIA and the Extreme-ultraviolet Imaging Spectrometer (EIS). With one thread on a $3.2 \mathrm{GHz}$ processor, it is able to compute well over $1000 \mathrm{DEMs} \mathrm{s}^{-1}$ for an example solar active region observed by AIA, and over 100 DEMs s$^{-1}$ in our most difficult test cases. Moreover, we anticipate that with straightforward optimizations (e.g., conversion of computationally intensive portions of the code to $\mathrm{C}$ and parallelization), the performance of the code will be increased to $\sim 10^{5}$ DEMs s$~^{-1}$ on a single workstation, sufficient to match AIA's real-time observing rate.

This paper describes the method, analyzes its fidelity, compares its performance and results with other DEM methods, and applies it to example solar data. We also touch on the limitations of the solar data to constrain the DEM being observed. It must be noted that the ability to recover the details of the original solar input DEM is limited, both because the number of channels are limited and because the temperature response functions are broad, due to the broad temperature-dependent emissivities of the spectral lines from which they are constructed. This is discussed in detail, for instance, in Craig \& Brown (1976) and Judge et al. (1997).

\section{DEM ALGORITHM}

\subsection{First Pass}

We wish to infer the DEM, a continuous function, from a small number of observed intensities which are the result of the convolution of the DEM with the instrument response functions. This problem is inherently ill-posed due to the limited number of response functions and the loss of information resulting from the convolution (i.e., Equation (1)). To resolve the ambiguity, we impose additional constraints on the DEM solution. The first is that the DEM can be expressed as a linear combination 
of some set of basis elements (e.g., a set of narrow temperature bins), $B_{j}(T)$, with coefficients $e_{j}$ (i.e., $\mathcal{E}(T)=\sum_{j} e_{j} B_{j}(T)$ ). The integral Equation (1) then becomes a matrix equation:

$$
\begin{aligned}
g_{n} & =\int R_{n}(T) \mathcal{E}(T) d T \\
& =\sum_{k}\left[e_{k} \int R_{n}(T) B_{k}(T) d T\right] \equiv \sum_{k} e_{k} \gamma_{n} A_{n k},
\end{aligned}
$$

where $\gamma_{n}$ are a set of normalization constants for the response functions, which we choose to be $\int R_{n}^{2}(T) d T$ (in conjunction with the instrument response basis discussed below, this results in a symmetric $A_{n k}$ matrix whose diagonal elements are unity).

If the number of basis elements is greater than number of instrument channels, the underconstrained inversion can be resolved by a singular value decomposition (SVD; Press et al. 2002), which picks the coefficient vector with the smallest magnitude (i.e., least-squared emission measure, EM). This is a sort of smoothness constraint, since a solution which has emission concentrated into narrow peaks will have greater total squared EM than a solution which spreads the emission as broadly as the data will allow. Such a solution will also tend to reduce non-physical negative emission, since negative EM is likely to require excess positive EM elsewhere in order to produce the observed intensities, resulting in relatively high total squared EM.

If the number of basis elements is equal to the number of instrument channels, it is straightforward to invert Equation (2) to find the DEM coefficients, $e_{k}$. In that case, however, the quality of the inversion is highly sensitive to choice of basis. In particular, many choices of basis lead to an inversion matrix (i.e., $A_{j k}^{-1}$ ) that has large negative entries, causing large negative values in the resulting DEMs.

The tendency of the inverse matrix to have large negative values is reduced, however, if $A_{j k}$ is diagonally dominant (i.e., each diagonal entry of the matrix is the largest one in its respective row/column). In particular, if we choose the basis functions to be the instrument response functions themselves, square-normalized, we obtain a symmetric $A_{j k}$ matrix whose diagonal entries are unity, with all off-diagonal entries less than one. This choice obviously restricts our attention to a small subspace of the set of possible functions $E(T)$. However, it is a natural subspace for $E(T)$, given the limitations imposed by the ill-posed inversion. Later, we will move our result out of this subspace by introducing the positivity constraint.

Remarkably, we find that this basis gives results identical to using a large number of narrow basis elements and an SVD (the Appendix demonstrates why this is the case). It therefore satisfies the SVD's minimum squared EM constraint, and we use it to compute a first pass DEM as a starting points for our DEM inversion. It reduces the number of operations required for each pixel to only $\sim N^{2}$ operations with $N$ instrument channels, increasing the speed of the inversion.

Even when using the response functions as a basis, however, we continue to compute $A_{i j}^{-1}$ using an SVD. This allows us to enforce a minimum condition $\left(\sim 10^{-12}\right.$, typically $\left.{ }^{2}\right)$ on the inversion, ensuring that round-off error (from $A_{i j}$ being nearly singular) does not cause ringing. In effect, this combines highly

\footnotetext{
2 This means the smallest singular value used to form the inverse matrix must be at least $10^{-12}$ times the largest singular value. See Press et al. (2002) for a detailed discussion.
}

similar channels (or linear combinations of channels, to be more precise) into a single channel rather than trying to fit them individually.

\subsection{Regularization}

Another constraint which DEM solutions must satisfy is a physically required positivity constraint. However, the first pass DEM inversion described in Section 2.1 can produce negative coefficients and therefore negative EM. This is particularly true when the errors in the observed intensities are significant, as the first-pass solutions exactly reproduce the input data and there is no guarantee that a set of noisy data intensities can be exactly reproduced by a purely positive DEM. To mitigate this issue, we once again seek to minimize the total squared EM, but we now allow the data values of the DEM, $g_{j}^{\prime} \equiv \sum_{k} e_{k} \gamma_{n} A_{n k}$, to deviate from the input data (i.e., $g_{j}$ ) at a specified $\chi^{2}$ level. This is a form of regularization, a standard technique in inverse problems (see Craig 1977; Craig \& Brown 1986; Hannah \& Kontar 2012), which we implement by seeking a new DEM that minimizes the sum

$$
\chi^{2}+\lambda \int[\mathcal{E}(T)]^{2} d T=\sum_{j} \frac{\left(\Delta g_{j}\right)^{2}}{\sigma_{j}^{2}}+\lambda \sum_{j k} e_{j} e_{k} A_{j k}
$$

where $\mathcal{E}(T)$ is the DEM described above, $\Delta g_{j} \equiv g_{j}-g_{j}^{\prime}$, and $\lambda$ is a Lagrange multiplier which we adjust so that the minimum occurs at the desired $\chi^{2}$ threshold, $\chi_{0}^{2}$. Note that the right-hand side of Equation (3) assumes we are using the basis of instrument response functions described in Section 2.1. Inverting Equation (2), we replace $e_{i}$ with $\Delta g_{i}$ (i.e., we let $\left.e_{j}=\sum_{k} A_{j k}^{-1}\left(g_{k}+\Delta g_{k}\right) / \gamma_{k}\right)$ :

$\chi^{2}+\lambda \int[\mathcal{E}(T)]^{2} d T=\sum_{j} \frac{\Delta g_{j}^{2}}{\sigma_{j}^{2}}+\lambda \sum_{j k}\left(g_{j}+\Delta g_{j}\right) \frac{A_{j k}^{-1}}{\gamma_{j} \gamma_{k}}\left(g_{k}+\Delta g_{k}\right)$.

For a given value of $\lambda$, this is minimized by a specific set of data corrections, $\Delta g_{j}(\lambda)$, which occur where the gradient of Equation (4) (with respect to $\Delta g_{j}$ ) is equal to zero. $\Delta g_{j}(\lambda)$ determines a corresponding $\chi^{2}(\lambda)$, and we use a bisection search to find the value of $\lambda, \lambda_{0}$, which has $\chi^{2}\left(\lambda_{0}\right)=\chi_{0}^{2}$. The regularized data corrections are then $\Delta g_{j}\left(\lambda_{0}\right)$, and the corresponding regularized data values are $g_{j}^{\prime}\left(\lambda_{0}\right)=g_{j}+$ $\Delta g_{j}\left(\lambda_{0}\right)$.

$\chi_{0}^{2}$ determines the regularization strength, and is chosen to be of order unity to ensure agreement with the data while avoiding overfitting. Our choice of this threshold is discussed near the end of Section 2.3. The new regularized DEM is simply the first-pass inversion applied to the new corrected data values.

Regularized solution of the DEM problem has recently been discussed by Hannah \& Kontar (2012), but our regularization is simpler and faster owing to the basis set used for the DEM. Since DEMs constructed from the instrument response functions have only a small number of basis elements, however, they remain liable to producing negative emission in cases with sharp features. The solution to this problem is discussed next.

\subsection{Enforcing Non-negativity}

Beginning with the regularized data, $g_{j}^{\prime}\left(\lambda_{0}\right)$, we remove the remaining negative emission via the following iterative process.

1. Zero the negative EM in the current DEM, $\mathcal{E}^{(n)}$, to create a new DEM, $\mathcal{E}_{+}^{(n)}$. At the zeroth iteration, this is the 
regularized DEM from Section $2.2\left(\mathcal{E}^{(0)}=\sum_{j} e_{j} B_{j}(T)=\right.$ $\left.\sum_{j k} B_{j}(T) A_{j k}^{-1} g_{j}^{\prime}\left(\lambda_{0}\right) / \gamma_{k}\right)$.

2. Compute the data intensities, $g_{j}^{+}=\int \mathcal{E}_{+}^{(n)}(T) R_{j}(T) d T$, corresponding to $\mathcal{E}_{+}^{(n)}$.

3. Take the difference between these data intensities and the original regularized data intensities, $\Delta g_{k}^{+} \equiv g_{j}^{\prime}\left(\lambda_{0}\right)-g_{j}^{+}$.

4. Compute correction DEM coefficients, $\Delta e_{j}=$ $\sum_{k} A_{j k}^{-1} \Delta g_{k}^{+} / \gamma_{k} . A_{j k}^{-1}$ is computed using an SVD at this step, and we enforce a relatively strong minimum condition number of $\sim 0.1$ (the minimum condition number is discussed in Section 2.1) to reduce ringing in the correction.

5. Subtract the corresponding DEM corrections, $\Delta \mathcal{E}^{(n)}$ from $\mathcal{E}_{+}^{(n)}$. By construction, this restores $\mathcal{E}^{(n+1)} \equiv \mathcal{E}_{+}^{(n)}-\Delta \mathcal{E}^{(n)}$ to agreement with the regularized data, but reintroduces some negative emission.

6. Repeat from step 1 until $g_{i}^{+}$matches $g_{j}^{\prime}\left(\lambda_{0}\right)$ to within the desired $\chi^{2}$.

By zeroing negative values in the first-pass DEM, we have created a DEM function that cannot be represented by the basis of instrument response functions. We therefore require a basis which more closely approximates a general function of a real number. We choose a basis of uniformly gridded triangle functions, defined by

$$
B_{j}^{\prime}(T)=\left\{\begin{array}{ll}
1-\frac{\left|T-T_{j}\right|}{\Delta T} & \text { if }\left|T-T_{j}\right|<\Delta T \\
0 & \text { otherwise }
\end{array},\right.
$$

where the grid centers, $T_{j}$, are separated by $\Delta T$. The first-pass DEM is mapped onto this basis prior to zeroing negative EM coefficients in step 1.

To further speed convergence of the iteration, we attempt a linear extrapolation at each step of the iteration. The extrapolation steps move the DEM vector further along the direction of the current iteration step, and are only accepted when they improve $\chi^{2}$.

We find that the optimal regularization strength (enforced via $\chi_{0}^{2}$ from Section 2.2), and minimum condition number strength in step 4 of the iteration, vary with input DEM and data quality, so we try multiple values of these parameters, beginning with a light regularization and small minimum condition number threshold and moving to stronger regularization and larger minimum condition number threshold. Typical values of these parameters are $[0.9,0.5,0.01]^{3}$ and $[0.01,0.05,0.1]$, for the regularization strengths and minimum condition numbers, respectively. In broad terms, the operation of the algorithm can then be described as follows.

1. Apply the light (e.g., 0.9) regularization to the data and compute the corresponding first-pass DEM.

2. Zero the negative emission in the first-pass DEM and compute the $\chi^{2}$ of the resulting modeled intensities with respect to the initial data. If $\chi^{2}$ exceeds the desired threshold, attempt to iterate away the negative emission, using the smallest minimum condition number threshold (e.g., 0.01), until the $\chi^{2}$ threshold is reached.

\footnotetext{
3 We define the regularization parameters to be $p$-values of the $\chi^{2}$ distribution (see, for instance, Chapter 15 of Press et al. 2002, where they are referred to as $Q$, and $P$ refers to their complement). With a regularization parameter of 0.9 , for instance, the $\chi_{0}^{2}$ of the regularized data with respect to the original data will correspond to a $p$-value of 0.9 .
}

3. If the iteration takes too long to reach the desired $\chi^{2}$ threshold, retry the first two steps with the next strongest regularization strengths and minimum conditions (e.g., 0.5 and 0.05 , respectively). Repeat until the $\chi^{2}$ threshold is reached, or each pair of regularization strengths and minimum conditions have been tried.

The final $\chi^{2}$ threshold is distinct from $\chi_{0}^{2}$, and in practice we choose it to be $10 \%$ larger than the largest $\chi_{0}^{2}$ value. For the 6 AIA channels, for instance, the values of $\chi_{0}^{2}$ corresponding to the regularization parameters of $[0.9,0.5,0.01]$ mentioned above (which can be computed in IDL via, for instance, chisqr_cvf $(0.01,6))$ would be $[2.20,5.34,16.81]$ (not reduced), and the final $\chi^{2}$ threshold would be $10 \%$ more than 16.81. Readers who would like more implementation details are encouraged to examine our code, which we have made publicly available at http://solar.physics.montana.edu/ plowman/firdems.tgz. We also intend to submit the code to SolarSoft in the near future.

\section{FIDELITY AND PERFORMANCE}

We have tested our DEM using a variety of example cases. The results are given in figures below, which show recovered DEMs, $\chi^{2}$ agreement with the data, and computational time. Note the following.

1. All reported $\chi^{2}$ have been reduced by dividing by the number of instrument channels being fit.

2. Unless otherwise noted, we use a $1 \mathrm{~s}$ exposure time for AIA, and $30 \mathrm{~s}$ for EIS. EIS pixels were assumed to be sampled to $2 \operatorname{arcsec}^{2}$.

3. AIA errors are assumed to be from read and shot noise alone, while EIS errors assume an additional $10 \%$ error from other sources.

4. We use the $94,131,171,193,211$, and $335 \AA$ AIA channels throughout.

5. EIS emissivities were computed assuming a density of $10^{9} \mathrm{~cm}^{-3}$, unless otherwise noted.

6. AIA response functions were computed by calling aia_get_response(/temp, /dn, /evenorm).

7. Computation times are for a single thread running on a $3.2 \mathrm{GHz}$ Intel Xeon processor, in IDL.

Wherever possible, we use $\log _{10}(T)$ as a temperature variable rather than $T$ (in Kelvin) itself. We believe this is the more natural parameter for the temperature, since we are interested in a temperature range spanning multiple orders of magnitude $\left(\sim 10^{5}-10^{7} \mathrm{~K}\right)$. Similarly, our DEMs are scaled per unit $\log _{10}(T)$, rather than per unit $T$. We believe this is the natural scaling when the DEMs are represented as functions of $\log _{10}(T)$, because the area under the DEM curves is then the EM. Rescaling to unit $T$ may be accomplished by dividing by $T \ln (10)$.

With few exceptions, we find that we are able to recover the test cases with good $\chi^{2}$ (reduced $\chi^{2}$ of one or two), that the recovered DEMs are a reasonable qualitative match to the input DEMs. Typical times for AIA DEMs are approximately $1 \mathrm{~ms}$, with some cases taking under $0.1 \mathrm{~ms}$. The test cases are as follows.

1. AIA inversion of lognormal DEMs with widths of 0.2 at selected temperatures (Figure 1). Average DN and errors for these examples are shown in Table 1.

2. AIA inversion of lognormal DEMs with widths of 0.5 at selected temperatures (Figure 2). 

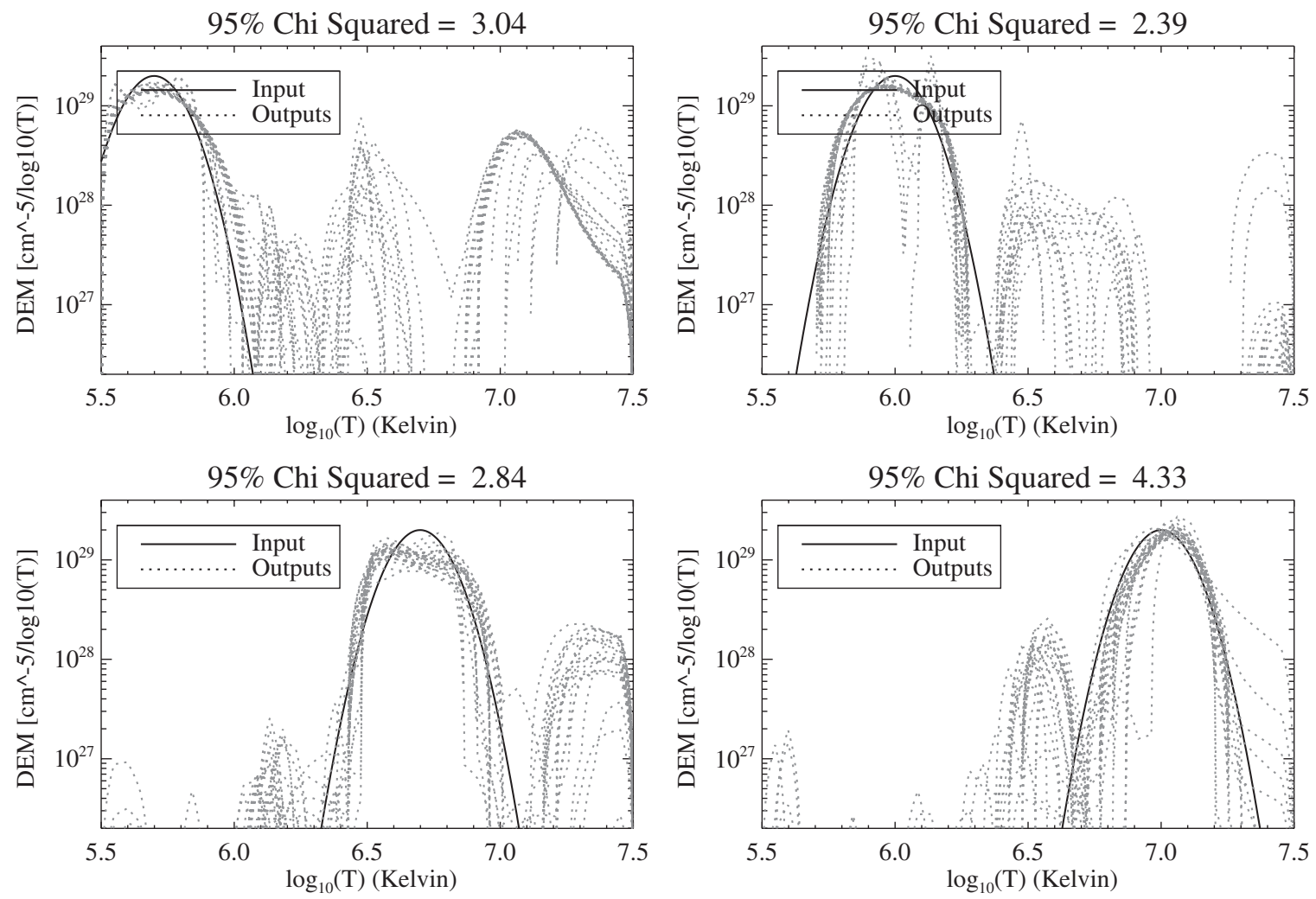

Figure 1. DEM inversions of lognormal simulated DEMs of width 0.2 using the six AIA EUV channels. The input DEM is shown by the solid line, while each dotted line is a DEM inversion with a distinct, random realization of the AIA read and shot noise. For each test case, $95 \%$ of solutions have $\chi^{2}$ less than or equal to the value listed in the corresponding title.
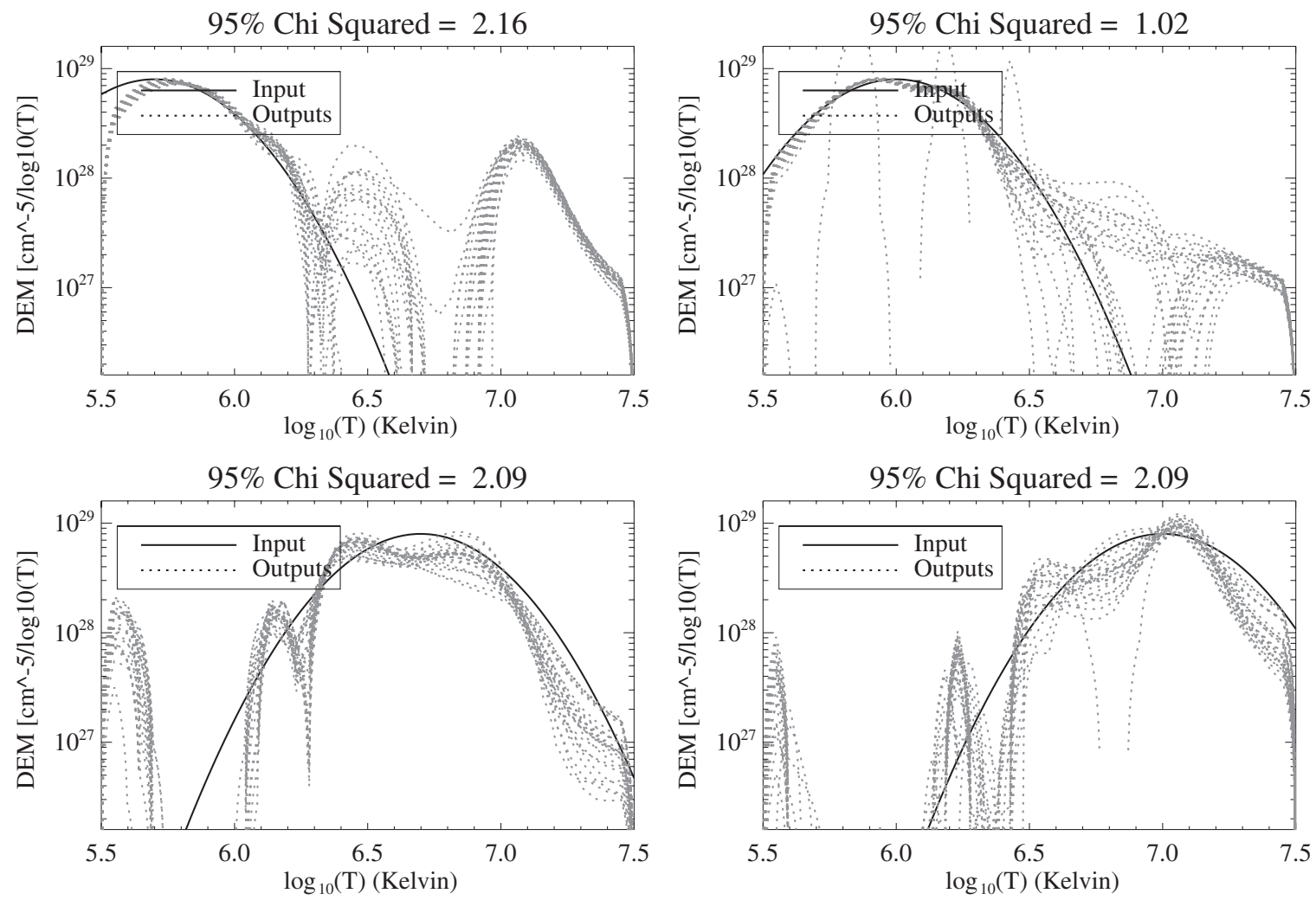

Figure 2. Same as Figure 1, but for a very broad DEM (width 0.5 ). 
Table 1

Average DN and Errors for the Example DEMs Shown in Figure 1

\begin{tabular}{lccccrrrr}
\hline \hline Channel & $5 \times 10^{5} \mathrm{~K} \mu$ & $\sigma$ & $1 \times 10^{6} \mathrm{~K} \mu$ & $\sigma$ & $5 \times 10^{6} \mathrm{~K} \mu$ & $\sigma$ & $1 \times 10^{7} \mathrm{~K} \mu$ & $\sigma$ \\
\hline A94 & 14.8 & 5.8 & 42.7 & 9.6 & 102.2 & 14.9 & 97.0 & 14.4 \\
\hline A131 & 1323.2 & 44.9 & 511.0 & 28.3 & 34.2 & 7.4 & 360.6 & 23.5 \\
\hline A171 & 25880.3 & 174.7 & 41617.8 & 221.4 & 51.3 & 7.9 & 18.3 & 4.8 \\
\hline A193 & 5711.0 & 76.2 & 14798.5 & 121.7 & 371.2 & 19.6 & 302.3 & 17.6 \\
\hline A211 & 829.8 & 28.1 & 2033.0 & 43.6 & 189.0 & 13.3 & 31.2 & 5.6 \\
\hline A335 & 177.2 & 10.3 & 206.6 & 11.1 & 119.4 & 8.5 & 31.5 & 4.5
\end{tabular}

Table 2

Average Line Intensities and Errors (in erg $\mathrm{cm}^{-2} \mathrm{~s}^{-1} \mathrm{sr}^{-1}$ ) for the Example DEMs Shown in Figure 3

\begin{tabular}{|c|c|c|c|c|c|c|c|c|}
\hline Channel & $5 \times 10^{5} \mathrm{~K}$ & $\sigma$ & $1 \times 10^{6} \mathrm{~K}$ & $\sigma$ & $5 \times 10^{6} \mathrm{~K}$ & $\sigma$ & $1 \times 10^{7} \mathrm{~K}$ & $\sigma$ \\
\hline Fe IX 188.497 & 5100.3 & 491.2 & 9992.8 & 1012.0 & 0.1 & 3.8 & -0.0 & 3.6 \\
\hline Fe x 184.537 & 1809.9 & 191.6 & 20101.2 & 2034.2 & 0.1 & 8.4 & -0.0 & 8.5 \\
\hline Fe XII 195.119 & 46.1 & 6.0 & 15559.9 & 1566.2 & 22.2 & 3.6 & -0.1 & 1.9 \\
\hline Fe xv 284.163 & 0.1 & 13.7 & 1460.8 & 150.4 & 9938.5 & 990.5 & 247.4 & 33.2 \\
\hline Fe XVI 262.976 & 0.1 & 6.4 & 11.6 & 7.5 & 1706.7 & 175.9 & 130.2 & 17.1 \\
\hline$M g \vee 276.579$ & 2892.5 & 290.3 & 42.1 & 9.4 & -0.0 & 6.3 & -0.0 & 6.4 \\
\hline Mg VI 270.394 & 6561.8 & 656.2 & 558.9 & 59.5 & -0.1 & 5.2 & 0.0 & 5.1 \\
\hline Mg VII 280.737 & 2775.7 & 280.9 & 1146.0 & 116.7 & 0.2 & 9.5 & 0.1 & 9.6 \\
\hline Fe IX 197.862 & 2847.9 & 287.8 & 6492.8 & 662.8 & -0.0 & 2.0 & -0.0 & 2.1 \\
\hline Fe XI 188.216 & 292.8 & 31.9 & 20087.0 & 2021.1 & 2.4 & 4.0 & 0.0 & 3.8 \\
\hline Fe XI 180.401 & 583.7 & 86.2 & 40824.1 & 4045.3 & 4.8 & 33.8 & 0.8 & 33.4 \\
\hline $\mathrm{S} \times 264.233$ & 7.8 & 6.4 & 936.1 & 98.5 & 3.4 & 6.4 & -0.2 & 6.1 \\
\hline Fe XII 192.394 & 14.7 & 3.3 & 4972.2 & 505.4 & 7.1 & 2.7 & -0.0 & 2.2 \\
\hline Fe XIII 202.044 & 2.0 & 7.0 & 3180.1 & 319.8 & 71.8 & 12.3 & 0.1 & 6.6 \\
\hline Fe XIII 203.826 & 0.9 & 12.8 & 2280.2 & 242.2 & 45.0 & 16.0 & 0.2 & 12.9 \\
\hline Fe XIV 270.519 & 0.1 & 5.1 & 411.1 & 44.2 & 142.7 & 17.1 & 1.0 & 5.1 \\
\hline S XIII 256.686 & -0.1 & 10.0 & 25.5 & 11.1 & 863.7 & 90.5 & 45.6 & 12.7 \\
\hline Ca XIV 193.874 & 0.0 & 2.0 & 0.2 & 2.0 & 376.7 & 39.0 & 5.6 & 2.3 \\
\hline Ca xv 200.972 & 0.1 & 4.5 & 0.0 & 4.4 & 443.6 & 47.9 & 18.2 & 5.7 \\
\hline Ca XVI 208.604 & -1.1 & 33.7 & -0.5 & 33.3 & 436.8 & 68.0 & 53.4 & 36.1 \\
\hline Ca XVII 192.858 & 0.0 & 2.1 & -0.1 & 2.1 & 1062.1 & 112.0 & 434.6 & 45.8 \\
\hline Si VII 275.361 & 12989.5 & 1329.2 & 6942.9 & 691.9 & 0.2 & 5.9 & 0.2 & 5.9 \\
\hline Si x 258.371 & 61.7 & 12.9 & 7228.0 & 725.3 & 12.1 & 9.4 & 0.2 & 8.6 \\
\hline Fe xIV 264.79 & 0.4 & 6.0 & 728.6 & 76.6 & 245.7 & 28.3 & 1.4 & 6.0 \\
\hline
\end{tabular}

Note. These lines are described in more detail in Warren et al. (2011).

3. EIS inversion of lognormal DEMs with widths of 0.2 at selected temperatures (Figure 3). Average line intensities and errors for these examples are shown in Table 2.

4. EIS inversion of lognormal DEMs with widths of 0.5 at selected temperatures (Figure 4).

5. AIA inversion of lognormal DEMs at temperatures ranging from $10^{5.5}$ to $10^{7.0} \mathrm{~K}$, for widths of 0.2 (Figure 5), 0.3 (Figure 6), 0.0001 (effectively isothermal; Figure 7), and 0.5 (Figure 8).

6. EIS inversion (using the 24 lines chosen by Warren et al. 2011) of lognormal DEMs at temperatures ranging from $10^{5.5}$ to $10^{7.0} \mathrm{~K}$, for widths of 0.2 (Figure 9), 0.3
(Figure 10), 0.0001 (effectively isothermal; Figure 11), and 0.5 (Figure 12).

7. AIA inversion of DEMs produced by summing five lognormal DEMs with randomly chosen centers, widths, and amplitudes (Figure 13).

8. EIS active region DEM from Warren et al. (2011; Figure 14). A density of $10^{9.5} \mathrm{~cm}^{-5}$ is assumed, to match their emissivities.

We find that narrow (compared with the temperature response functions in question) DEMs are the most difficult for our method to reconstruct, in terms of obtaining good $\chi^{2}$ (i.e., $\chi_{R}^{2} \sim 1$ ) without negative emission. This can be seen by comparing Figures $7,5,6$, and 8 (for AIA), or Figures 9 

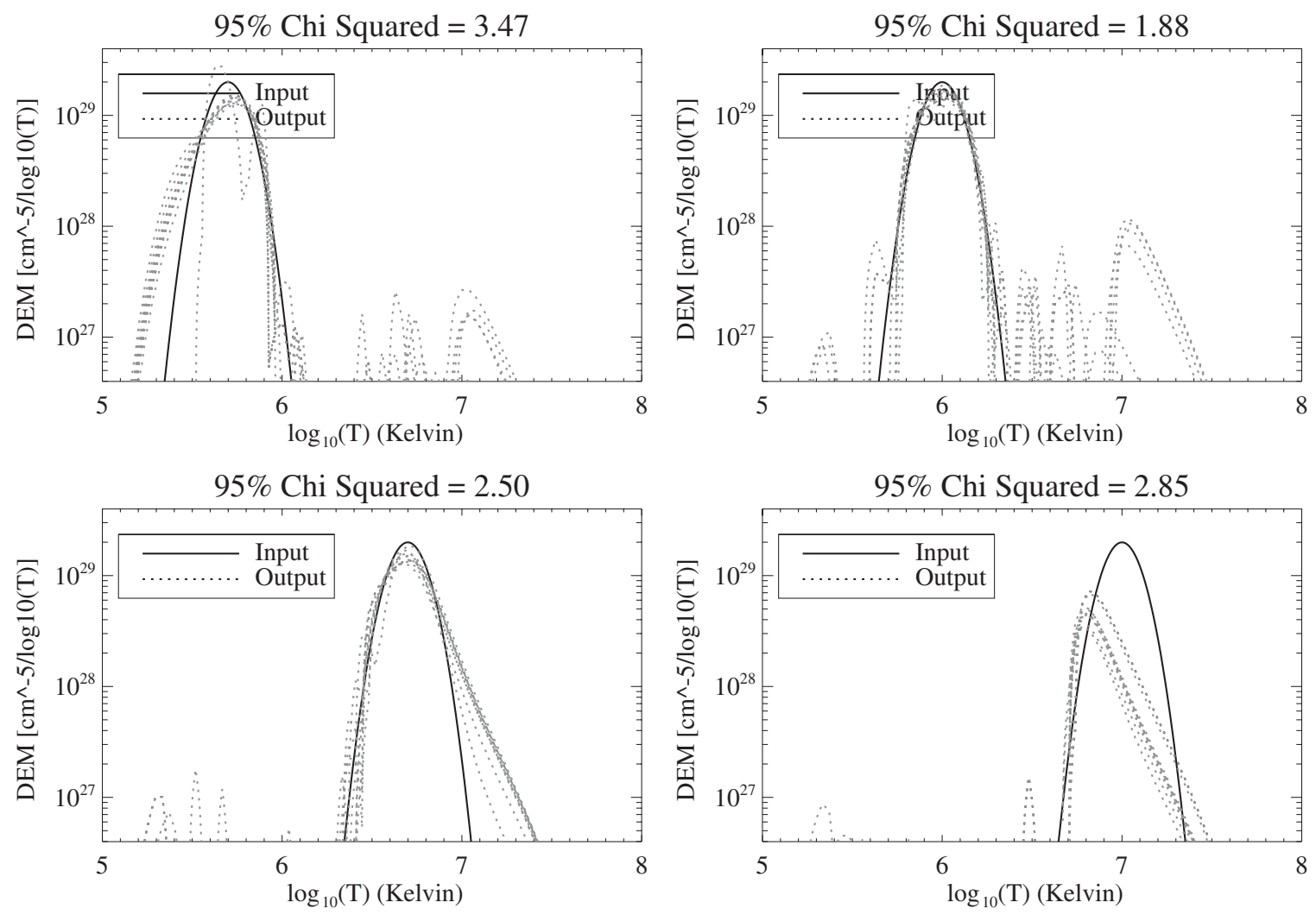

Figure 3. Same as Figure 1, but for the 24 EIS lines used in Warren et al. (2011).
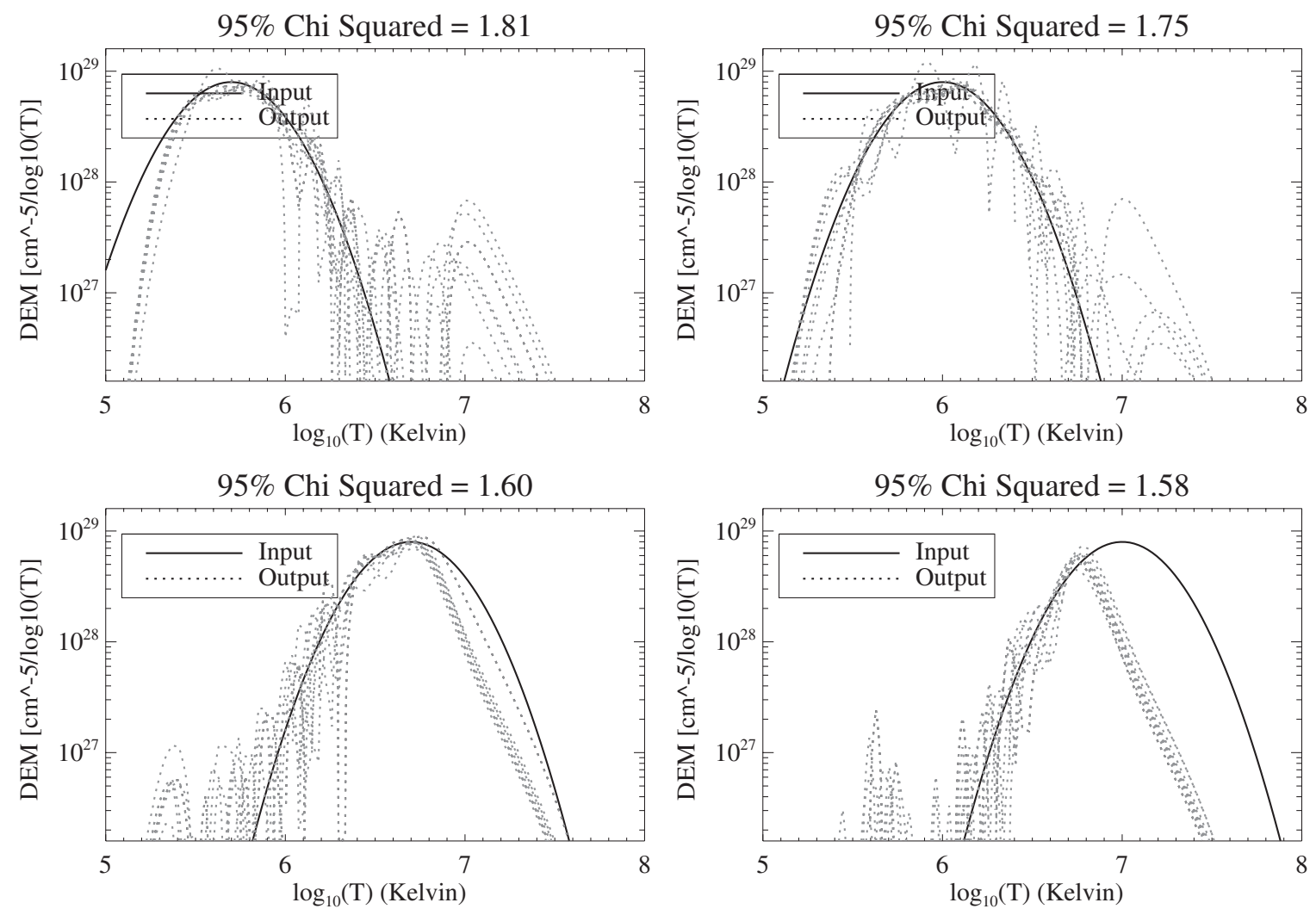

Figure 4. Same as Figure 2, but for the 24 EIS lines used in Warren et al. (2011). 

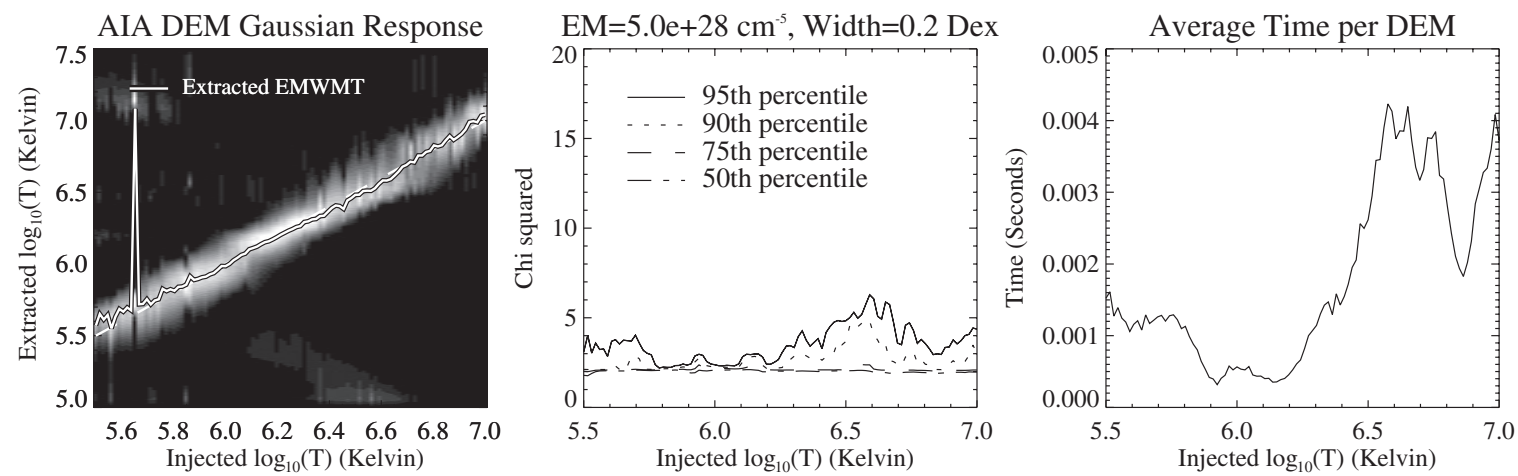

Figure 5. AIA response to lognormal DEMs of width 0.2 and total EM $5.0 \times 10^{28} \mathrm{~cm}^{-5}$ at temperatures from 5.5 to 7.0 dex. Left: the recovered DEMs-each vertical slice of this plot is a DEM-like one of the output curves of Figure 1, at the temperature of the corresponding $x$-axis value. The solid lines on left show emission measure weighted median temperature (EMWMT); note that the intensity scale in this figure is linear. Center: $\chi^{2}$ percentiles resulting from repeated MC trials of the read and shot noise, at each temperature. Right: the average time per DEM at each temperature.
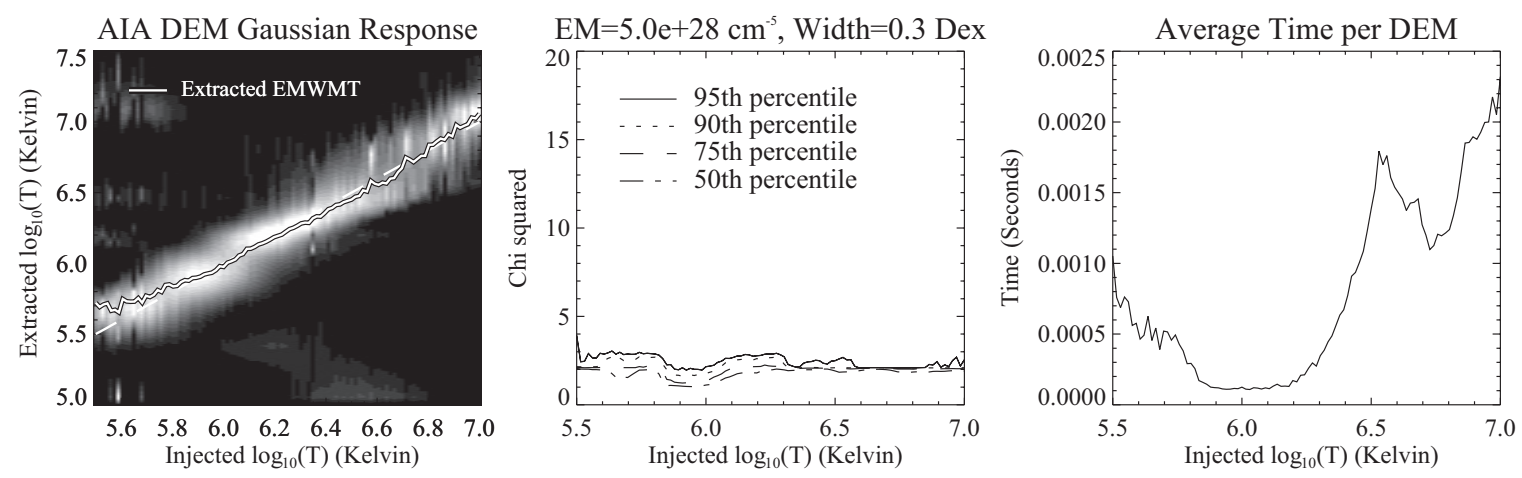

Figure 6. Same as Figure 5, but for lognormal DEMs of width 0.3 .
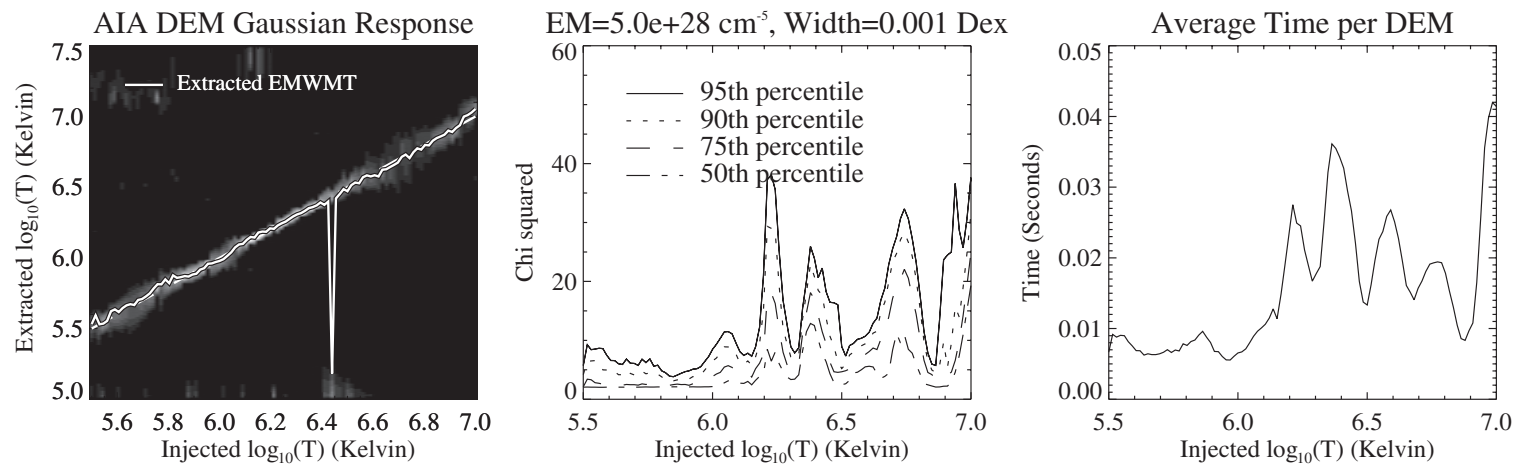

Figure 7. Same as Figure 5, but for effectively isothermal DEMs (width 0.001).
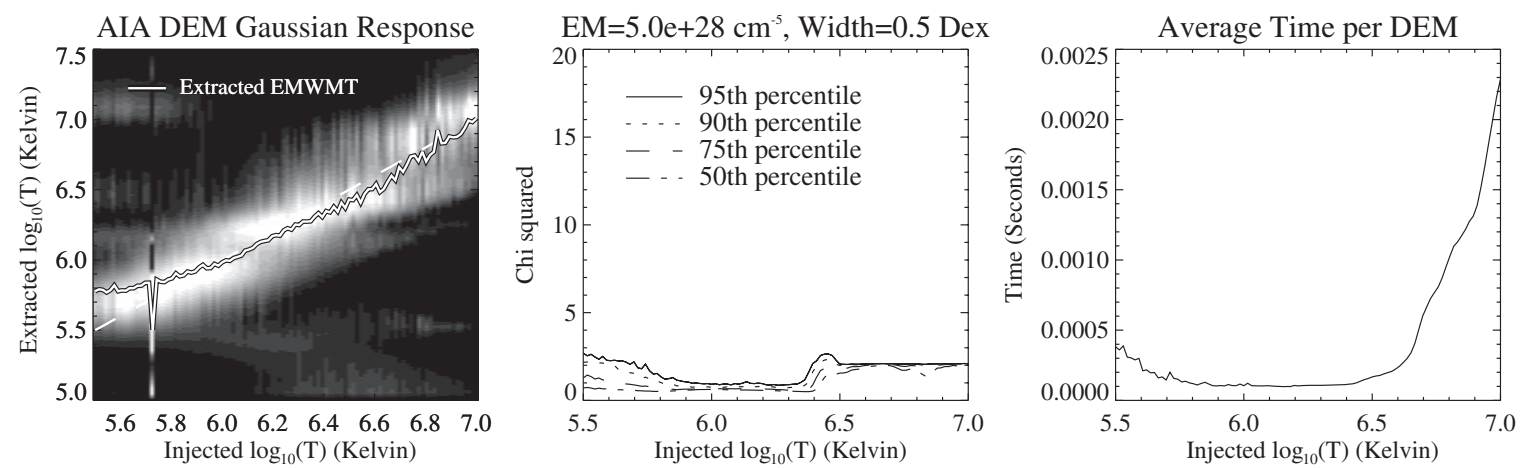

Figure 8. Same as Figure 5, but for very broad DEMs (width 0.5). 

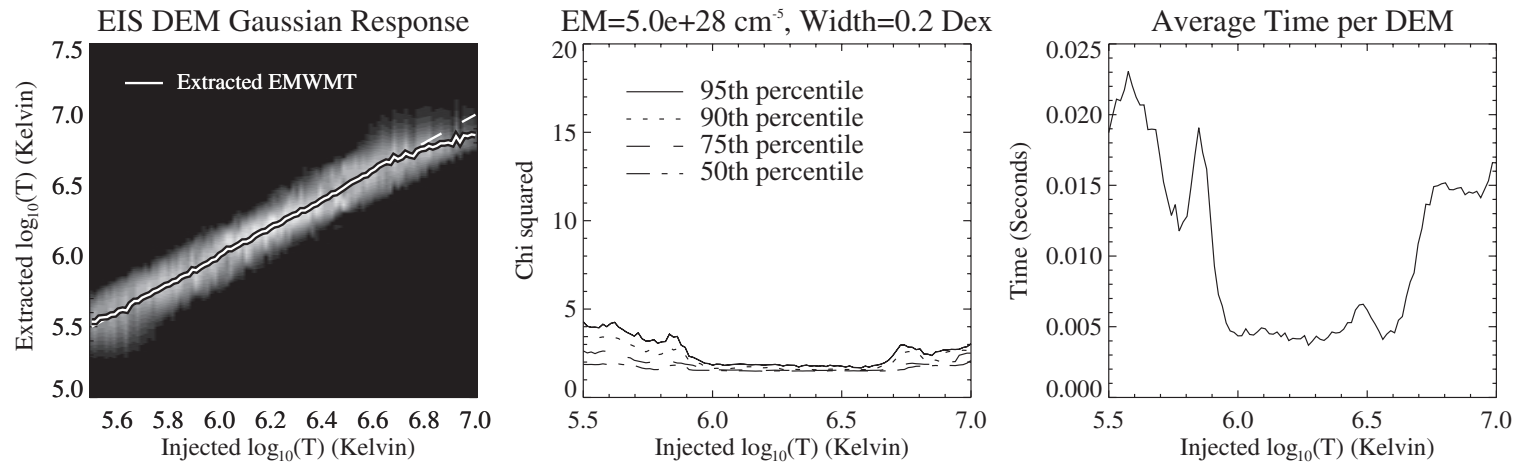

Figure 9. EIS response to lognormal DEMs of width 0.2 and total EM $5.0 \times 10^{28} \mathrm{~cm}^{-5}$ at temperatures from 5.5 to 7.0 dex. Solid lines on left show emission measure weighted median temperature (EMWMT). The 24 spectral lines from Warren et al. (2011) were used.
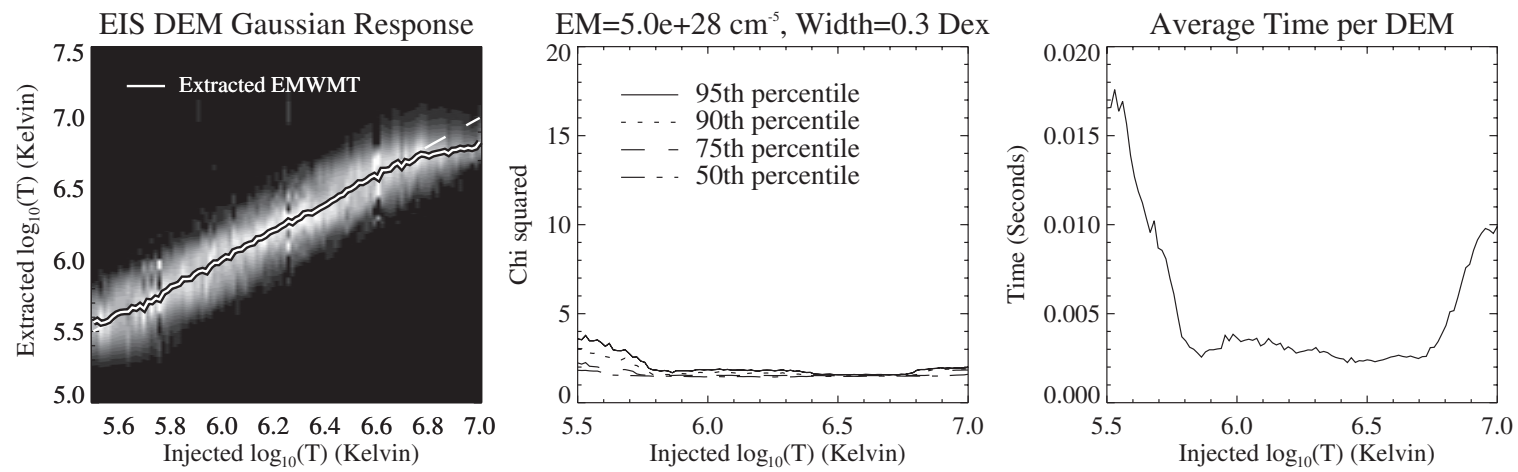

Figure 10. EIS response to lognormal DEMs of width 0.3 and total EM $5.0 \times 10^{28} \mathrm{~cm}^{-5}$ at temperatures from 5.5 to 7.0 dex. Solid lines on left show emission measure weighted median temperature (EMWMT). The 24 spectral lines from Warren et al. (2011) were used.
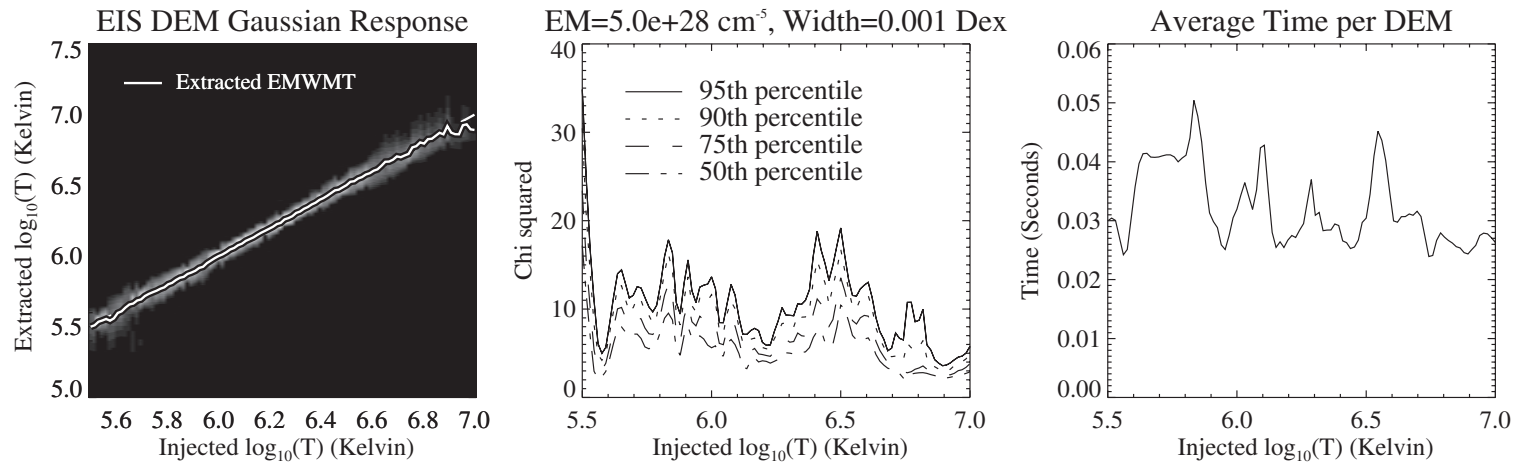

Figure 11. EIS response to effectively isothermal lognormal DEMs (width 0.001) and total EM $5.0 \times 10^{28} \mathrm{~cm}^{-5}$ at temperatures from 5.5 to 7.0 dex. Solid lines on left show emission measure weighted median temperature (EMWMT). The 24 spectral lines from Warren et al. (2011) were used.
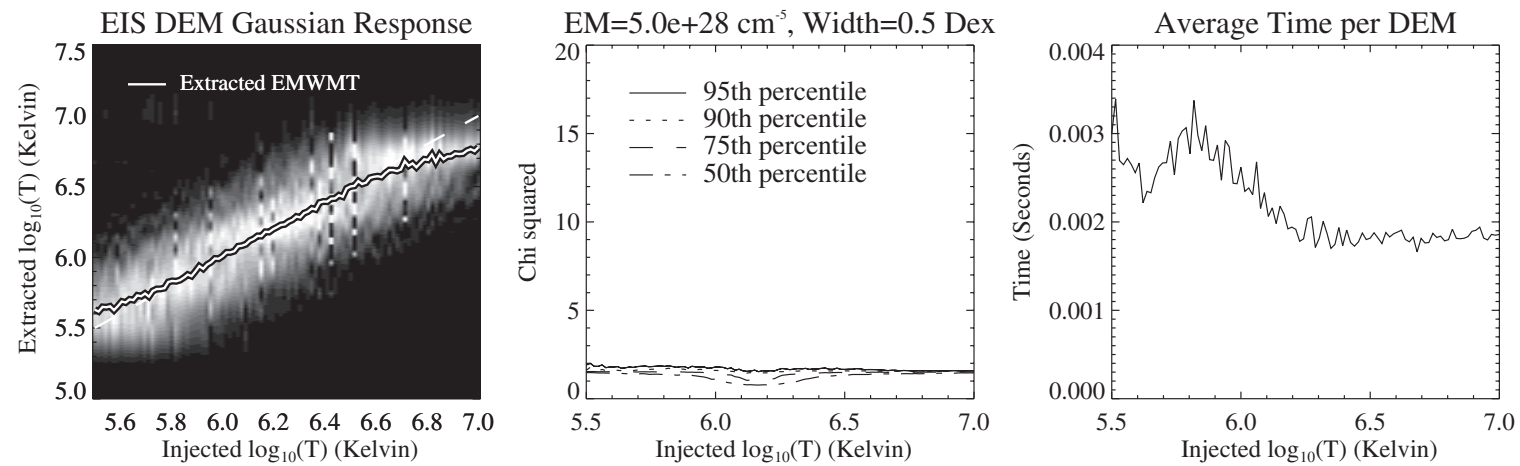

Figure 12. EIS response to lognormal DEMs of width 0.5 and total EM $5.0 \times 10^{28} \mathrm{~cm}^{-5}$ at temperatures from 5.5 to 7.0 dex. Solid lines on left show emission measure weighted median temperature (EMWMT). The 24 spectral lines from Warren et al. (2011) were used. 

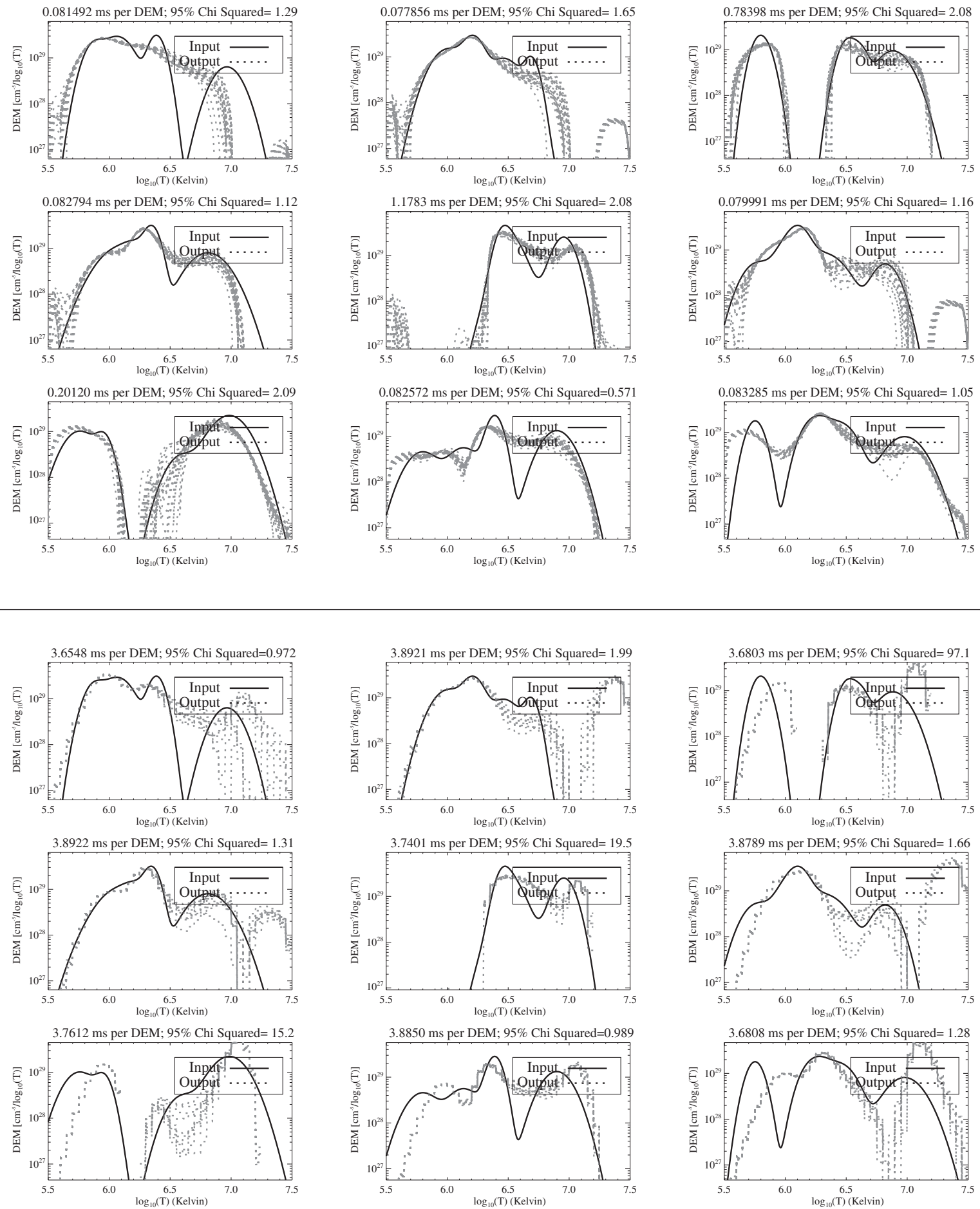

Figure 13. AIA inversion of DEMs produced by summing five lognormal DEMs with randomly chosen centers, widths, and amplitudes. Center locations are uniformly distributed between $10^{5.75}$ and $10^{7}$, widths between 0.1 and 0.3 , and total EM between $5 \times 10^{27} \mathrm{~cm}^{-5}$ and $5 \times 10^{28} \mathrm{~cm}^{-5}$. Top: results from our fast DEM method. Bottom: same for Hannah \& Kontar (2012) regularized DEM method, without positivity constraint.

and 10 (for EIS). For AIA, there is somewhat more difficulty in recovering narrow DEMs at temperatures above $10^{6.5} \mathrm{~K}$, but for all of the non-isothermal cases, acceptable $\chi^{2}$ were achieved for the majority of noise realizations. Despite their relatively poor $\chi^{2}$, these reconstructed DEMs are well behaved and localized at the center of the injected DEM; the median $\log _{10}(T)$ is recovered to within 0.1 between $\log _{10}(T) \approx 5.8$ and 7.0 .

For the isothermal case (Figure 7), recovering acceptable $\chi^{2}$ is problematic at some temperatures, but the recovered DEMs are very well localized around the injected temperatures. The difficulty in achieving good $\chi^{2}$ is absent in cases where the emission is not concentrated near a single temperature, as can be seen in Figures 2, 8, and 13. Qualitatively, the broad DEM reconstructions look somewhat worse than for the narrow DEMs - positivity is a weak constraint in this case because of the broad input DEM, and the regularization (enforced chiefly by our initial choice of basis, in this case) has a strong influence on the reconstruction. In all non-isothermal cases, the average time to compute a DEM for AIA was under $10 \mathrm{~ms}$, and less than $0.1 \mathrm{~ms}$ for some cases. 

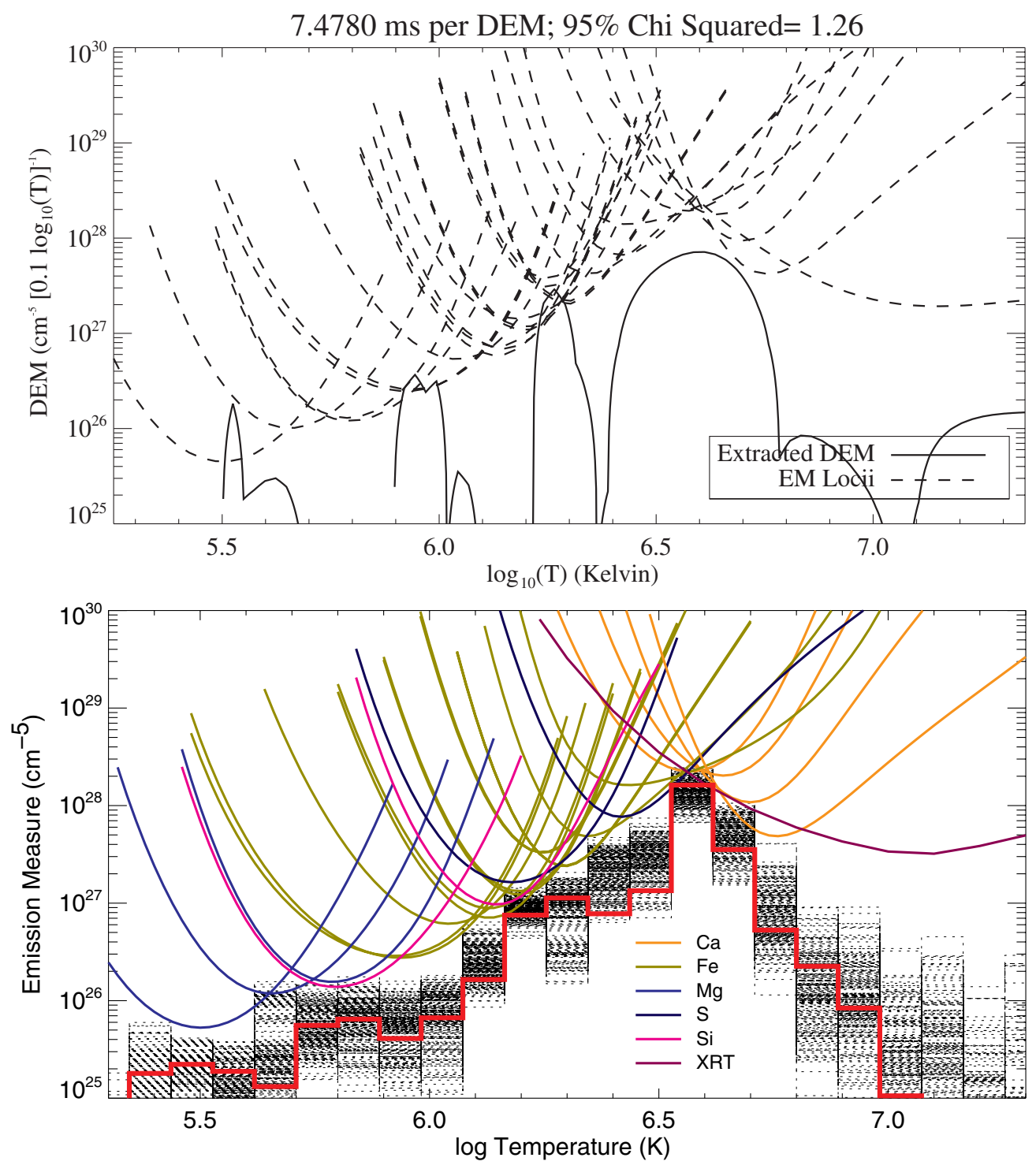

Figure 14. Comparison of our DEM results (top) with Warren et al. (2011; Figure 4) MCMC DEM results (bottom). We include their factor of 1.7 tweak to the coronal $\mathrm{Mg}$ abundance. The DEMs matched the data with $\chi^{2}$ of order unity.

(A color version of this figure is available in the online journal.)

In the case of EIS, temperatures greater than $10^{6.8} \mathrm{~K}$, above the highest temperature peak of the spectral lines used, are the most challenging. The median temperatures are accurately (i.e., $\left.\delta \log _{10}(T) \lesssim 0.1\right)$ recovered over a range of $\log _{10}(T), 5.6-6.8$. Due to the larger number of channels, the computational time is somewhat longer, at $\sim 10 \mathrm{~ms}$ for a narrow lognormal distribution.

In general, the primary peak of the injected test DEMs are well recovered, although the reconstructions also contain secondary artifacts, with relatively low EM, at other temperatures. This is more pronounced in the AIA inversions; it has fewer channels, some with multiple peaks, so the inversion problem is less well constrained. The 0.5 MK reconstructions in Figures 1 and 2 (upper left panel), for instance, contain a noticeable artifact at 10 MK. This can be attributed to the fact that the AIA $131 \AA$ channel has peaks at both of those temperatures, while the other channels are relatively insensitive there. The comparatively poor recovery of high-temperature emission from EIS data (e.g., the bottom right panel of Figure 4) is due to the selected spectral lines lacking sensitivity to emission at higher temperatures (the hottest line in the selection, CaXVII 192.858, has peak sensitivity at $\sim 6 \mathrm{MK})$.

Figure 13 compares our DEM inversions (top set) with those of the optimized Hannah \& Kontar (2012) (bottom set) "demmap" code. We ran their code without its positivity constraint option, finding better $\chi^{2}$ were achieved by simply zeroing the negative emission. The test DEMs are randomly generated multimodal distributions, as observed by AIA. Both codes were run in a single thread on the $3.2 \mathrm{GHz}$ processor mentioned above. The results are qualitatively quite similar, although the Hannah \& Kontar method appears to produce some spurious high-temperature emission. For these test cases, our DEM method is faster at $\sim 10^{-4} \mathrm{~s}$ for most DEMs compared with $3.7 \times 10^{-3} \mathrm{~s}$ (without positivity constraint) for the Hannah \& Kontar DEM. Our method also appears to produce reasonable $\chi^{2}$ with zero negative emission more consistently than the Hannah \& Kontar DEM inversions.

Figure 14 compares our DEM results with the PINTofALE MCMC results reported in Figure 4 of Warren et al. (2011). These results include all 24 EIS lines used by Warren et al., 

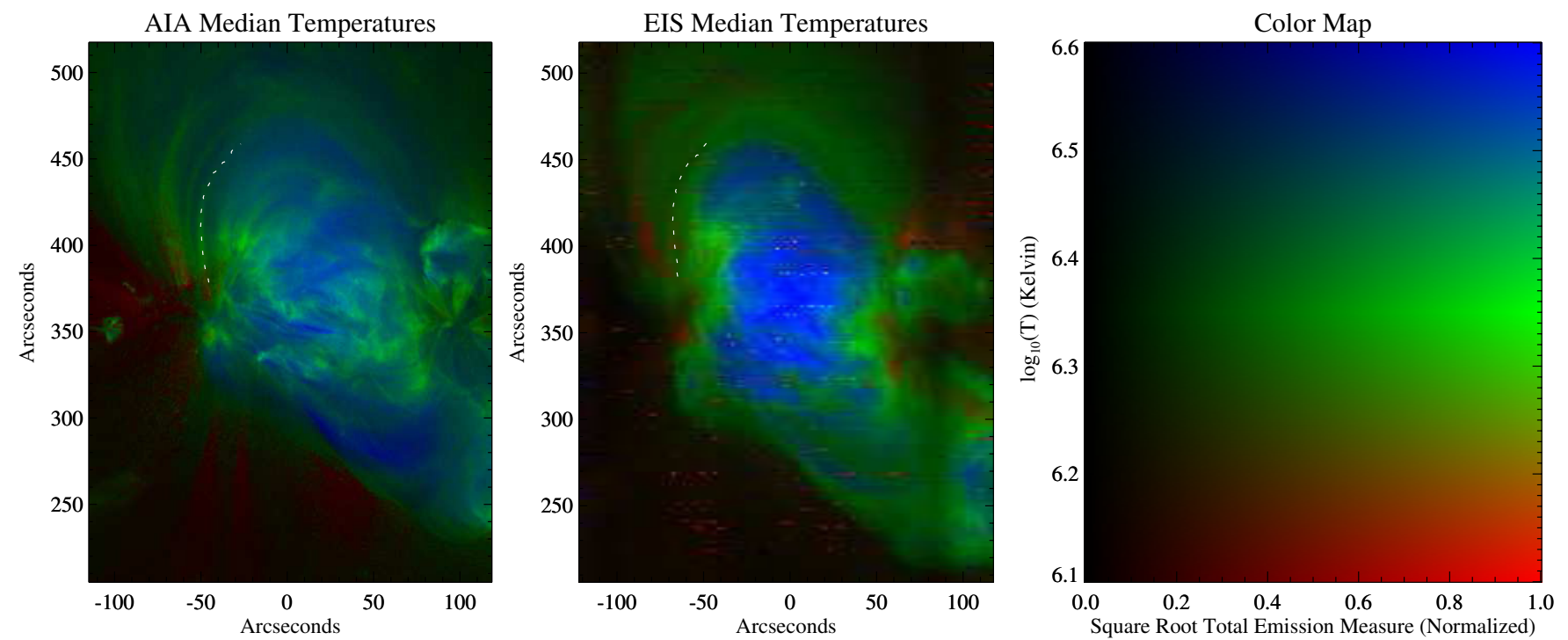

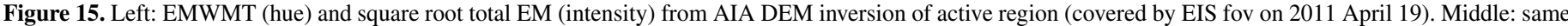
for EIS. Right: color scale for middle and left plots. Corresponding representative intensity images are shown in Figure 16.
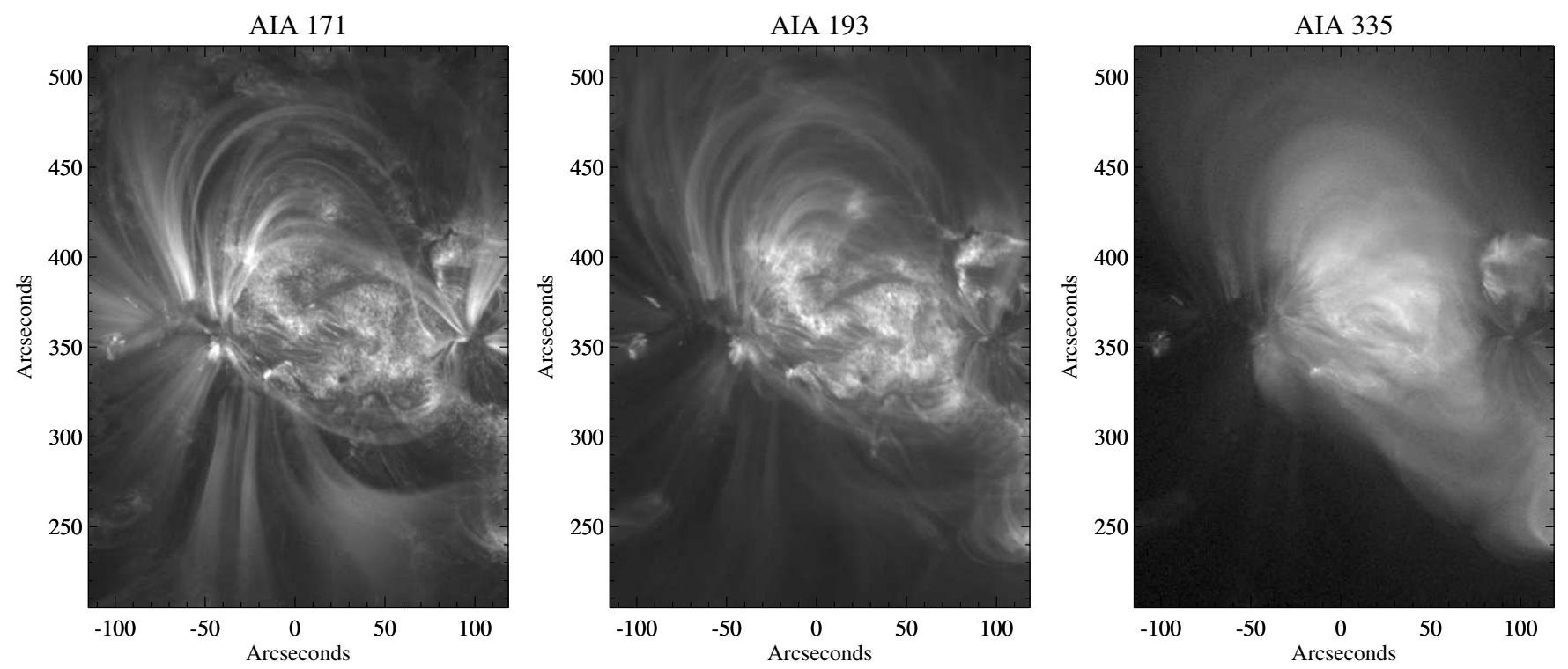

Figure 16. Representative intensity images (AIA 171, 193, and $335 \AA$ channels), square root scaled, for Figure 15.

with their factor of 1.7 tweak to the Mg intensities, along with the XRT Open/Al-thick filter. Our DEM curve differs than that of Warren et al. (2011). In particular, although our DEM also peaks at $\log _{10}(T) \approx 6.6$, the peak is broader, with a full width at half-maximum of $\sim 0.3$ versus $\sim 0.1$, and considerably lower in amplitude $\left(\sim 5 \times 10^{27} \mathrm{~cm}^{-5}\right.$ versus $\left.\sim 2 \times 10^{28} \mathrm{~cm}^{-5}\right)$. Despite this difference, we obtain a very reasonable reduced $\chi^{2}$ of 1.26 , which is fully consistent with the data.

\subsection{Example Solar DEM Analysis}

Finally, we show example DEM analysis of solar data. Figure 15 shows emission weighted median temperature (EMWMT) and total EM for an active region observed by EIS and AIA. The data cover the area of the large raster taken by EIS at around 01:30 on 2011 April 19. They are centered $\sim 350$ arcsec above disk center, with an approximately $230 \times 510$ arcsec field of view. The EIS data used fits to a set of five iron lines: Fe IX 188.497, Fe X 184.537, Fe XII 195.119, Fe XV 284.163, and Fe XVI 262.976.
The average time per AIA DEM was approximately $0.13 \mathrm{~ms}$, and we achieve reasonable $\chi^{2}$ for over $95 \%$ of the AIA DEMs (the 95th percentile $\chi^{2}$ for EIS is 4.7 ). The active region plots in Figure 15 are qualitatively quite similar, suggesting that the results give useful insight into the underlying solar temperature distribution.

We also show DEMs along the loop segment indicated by the dashed line in Figure 15. Figure 17 shows DEMs along the length of the loop segment (solid lines in Figure 18), as well as equivalent sets of DEMs offset to either side of the loop (dotted lines in Figure 18). Once again, the DEMs found by AIA and by EIS are reasonably consistent, and both EIS and AIA inversions achieved good $\chi^{2}$ for over $95 \%$ of the points along the loop.

The DEMs shown along the loop axis are not made from background subtracted data, and they show little difference from the offset DEMs, which indicates that the loop emission has been swamped out by the bright background. In an upcoming work, we compute background-subtracted DEMs for this loop and 

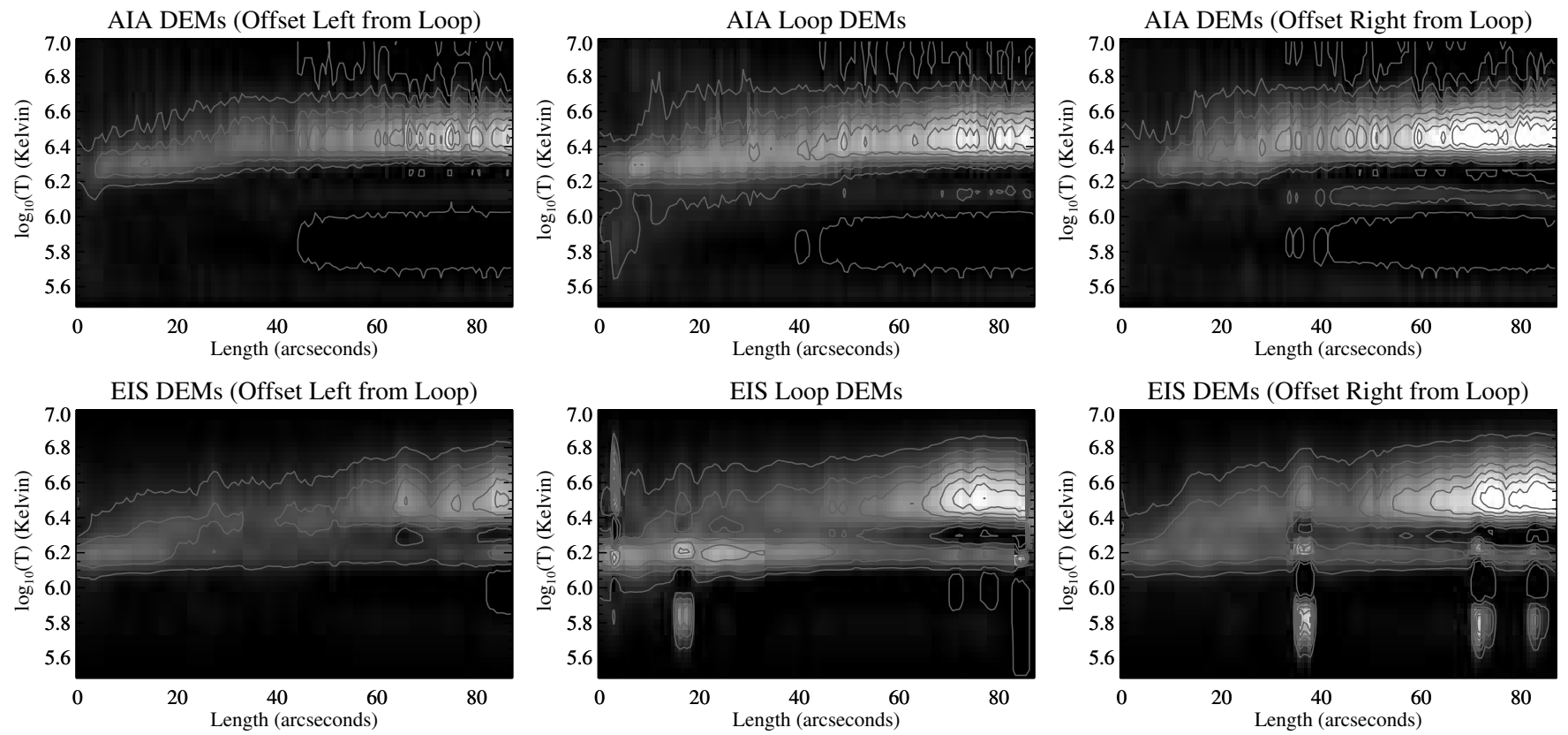

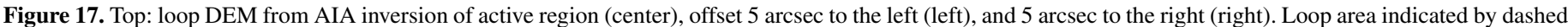
line in Figure 15, or in more detail in Figure 18. Bottom: same for EIS.
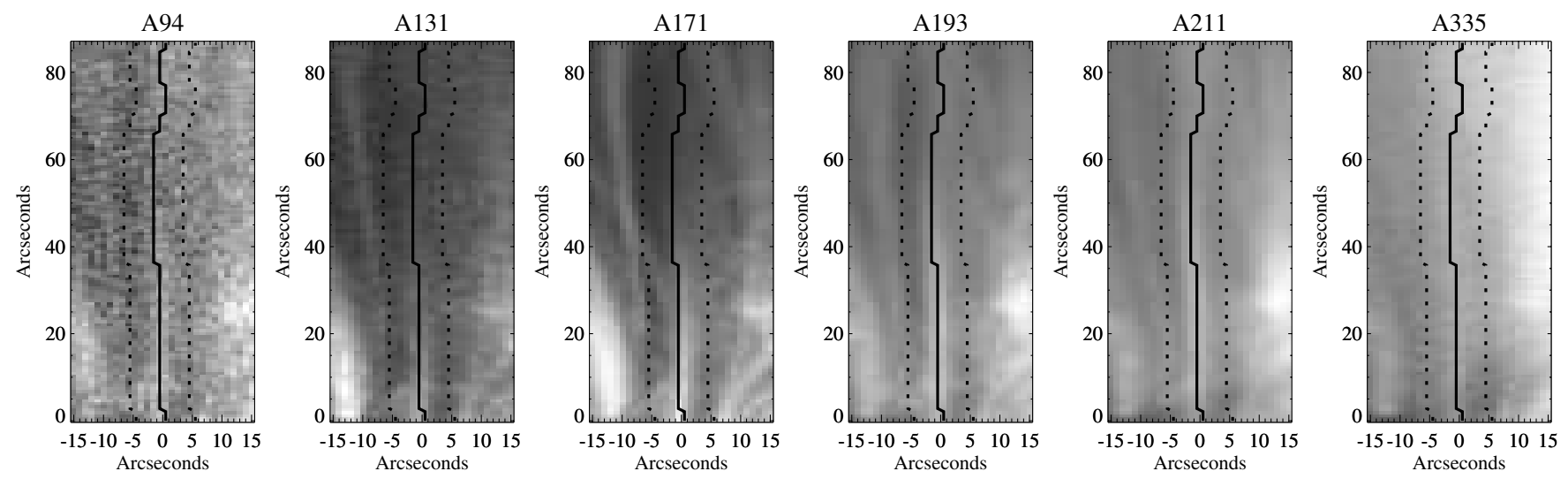

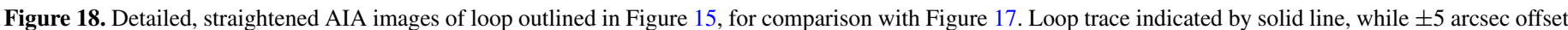
background traces are indicated by dotted lines.

compare its temperature and density profile to a set of analytic strand heating models.

\section{CONCLUSIONS}

We have demonstrated a method for fast reconstruction of DEM distributions using coronal data from instruments such as EIS and AIA. This DEM method achieves reduced chi-squared of order unity with no negative emission in all but a few test cases. The most difficult test cases are narrow DEMs at high $\left(>10^{6.5} \mathrm{~K}\right)$ temperatures for AIA, and temperature regions with little spectral coverage for EIS. Even for the high-temperature AIA cases, we achieve reasonable $\chi^{2}$ for the majority $(\sim 75 \%)$ of noise realizations. Qualitatively, the reconstructed DEMs match the input DEMs well, although the ability to recover finer details of the input DEMs is inherently limited.

The data, particularly for AIA, do not constrain the fine details of the DEMs, and the test cases investigated here do result in some artifacts (see Figure 1, for instance) in the inversions produced by our algorithm. The fact that the recovered $\chi^{2}$ are good is evidence of the ill-posedness of the DEM inversion problem; the data do not discriminate between the input DEM and the reconstructions, despite their differences. Any inversion algorithm must make choices to resolve these ambiguities, which can lead to inaccurate reconstructions of the actual DEM. Our algorithm is no different, and users must carefully consider which reconstruction method is most suited to their purposes. However, the test cases demonstrate that our algorithm does a reasonable job of recovering the dominant EM in the input DEMs. This, combined with its high performance, make it well suited to general use.

Our DEM method is fast enough to study the dynamics of the coronal temperature distributions in real time. For AIA data, the worst cases considered take under $10 \mathrm{~ms}$, and some test cases execute in less than $0.1 \mathrm{~ms}$. For the whole disk, at full AIA resolution using all six coronal EUV channels, we expect less than $\sim 1 \mathrm{hr}$ with the code as currently implemented. We plan to convert the computationally intensive parts of the code to $\mathrm{C}$ and rewrite them to take advantage of multithreading, which should offer a factor of $\sim 100$ 
performance gain on an eight-core workstation. This would reduce the time for a set of full-resolution, full-disk AIA DEMs to under $\sim 1$ minute, and achieve the objective of matching the AIA observing rate in real time. The software is available online at http://solar.physics.montana.edu/plowman/firdems.tgz, and will be submitted to SolarSoft in the near future.

We also applied our DEM reconstruction to solar data, an active region observed by EIS and AIA on 2011 April 19. We found no difficulty achieving reduced $\chi^{2}$ of order unity with no negative emission, and the average time per DEM was approximately $0.13 \mathrm{~ms}$, or $44 \mathrm{~s}$ for the entire active region at full AIA resolution. We find a relatively hot active region core with median temperature of around $3 \mathrm{MK}$, surrounded by cooler emission with median temperature of around $2 \mathrm{MK}$. We also plotted DEMs as a function of length along a coronal loop segment visible in the active region. These DEMs are dominated by a relatively bright background, making it unclear what emission is associated with the loop; background subtraction of the data will be necessary to isolate the loop emission from its surroundings.

\section{APPENDIX}

\section{EQUALITY OF SVD-DERIVED AND INSTRUMENT RESPONSE BASIS DEMs}

The fundamental equation we want to invert is the following:

$$
g_{j}=\int R_{j}(T) \mathcal{E}(T) d T
$$

Without loss of generality, we can represent this integral in matrix form:

$$
\boldsymbol{g}=\mathbf{R} \boldsymbol{E},
$$

where the elements of the $n \times m$ (for $n_{c}$ channels and $n_{t}$ temperature bins) response matrix $\mathbf{R}$ are $R_{j}\left(T_{k}\right)$, and the elements of the $n_{t}$-element DEM vector $\boldsymbol{E}$ are $\mathcal{E}\left(T_{j}\right) \Delta T$. The singular value decomposition of $\mathbf{R}$ is

$$
\mathbf{R}=\mathbf{U S V}^{\top}
$$

where $\mathbf{U}$ is an $n_{c} \times n_{c}$ unitary matrix, $\mathbf{V}$ is an $n_{t} \times n_{t}$ unitary matrix, and $\mathbf{S}$ is the $n_{c} \times n_{t}$ diagonal matrix of the singular values of $\mathbf{R}$. The SVD can be used to estimate $\boldsymbol{E}$ by computing the pseudo-inverse of $\mathbf{R}, \mathbf{R}^{+} \equiv \mathbf{V S}^{+} \mathbf{U}^{\top}$ :

$$
\boldsymbol{E}^{\mathrm{SVD}}=\mathbf{R}^{+} \boldsymbol{g}
$$

where $\mathbf{S}^{+}$is defined in terms $\mathbf{S}$ by replacing each nonzero diagonal entry with its reciprocal and transposing. We wish to show that this is identical to the following DEM inversion, computed using the instrument response functions as a basis:

$$
\boldsymbol{E}^{\mathrm{IR}}=\mathbf{R}^{\top} \boldsymbol{e}
$$

The $n_{c}$-element vector of coefficients $\boldsymbol{e}$ is obtained from $\boldsymbol{g}$ by plugging $\boldsymbol{E}^{\mathrm{IR}}$ into Equation (A2) and inverting

$$
\boldsymbol{e}=\left[\mathbf{R} \mathbf{R}^{\top}\right]^{-1} \boldsymbol{g} .
$$

Expanding $\left[\mathbf{R} \mathbf{R}^{\top}\right]^{-1}$ in terms of the SVD of $\mathbf{R}$, we find that

$$
\begin{aligned}
{\left[\mathbf{R R}^{\top}\right]^{-1} } & =\left[\mathbf{U S V} \mathbf{V}^{\top} \mathbf{V} \mathbf{S}^{\top} \mathbf{U}^{\top}\right]^{-1} \\
& =\left[\mathbf{U S S} \mathbf{S}^{\top} \mathbf{U}^{\top}\right]^{-1}=\mathbf{U}\left(\mathbf{S}^{+}\right)^{\top} \mathbf{S}^{+} \mathbf{U}^{\top},
\end{aligned}
$$

where the last equality follows directly from the unitarity of $\mathbf{U}$ and the definition of $\mathbf{S}^{+}$. We then obtain

$$
\begin{aligned}
\boldsymbol{E}^{\mathrm{IR}} & =\mathbf{R}^{\top}\left[\mathbf{R} \mathbf{R}^{\top}\right]^{-1} \boldsymbol{g}=\mathbf{V} \mathbf{S}^{\top} \mathbf{U}^{\top}\left(\mathbf{U}\left(\mathbf{S}^{+}\right)^{\top} \mathbf{S}^{+} \mathbf{U}^{\top}\right) \boldsymbol{g} \\
& =\mathbf{V} \mathbf{S}^{+} \mathbf{U}^{\top} \boldsymbol{g}=\mathbf{R}^{+} \boldsymbol{g} .
\end{aligned}
$$

Therefore, $\boldsymbol{E}^{\mathrm{IR}}=\boldsymbol{E}^{\mathrm{SVD}}$, as claimed.

\section{REFERENCES}

Craig, I. J. D. 1977, A\&A, 61, 575

Craig, I. J. D., \& Brown, J. C. 1976, A\&A, 49, 239

Craig, I. J. D., \& Brown, J. C. 1986, Inverse Problems in Astronomy: A Guide to Inversion Strategies for Remotely Sensed Data (Bristol: Hilger)

del Zanna, G., \& Mason, H. E. 2005, AdSpR, 36, 1503

Hannah, I. G., \& Kontar, E. P. 2012, A\&A, 539, A146

Judge, P. G., Hubeny, V., \& Brown, J. C. 1997, ApJ, 475, 275

Kashyap, V., \& Drake, J. J. 1998, ApJ, 503, 450

Lemen, J. R., Title, A. M., Akin, D. J., et al. 2012, SoPh, 275, 17

Press, W., Teukolsky, S., Vetterling, W., \& Flannery, B. 2002, Numerical Recipes in C: The Art of Scientific Computing (Cambridge: Cambridge Univ. Press) Warren, H. P., Brooks, D. H., \& Winebarger, A. R. 2011, ApJ, 734, 90 